
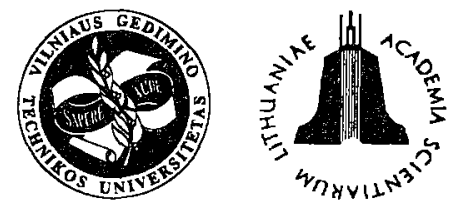

ISSN 1648-4142 TRANSPORT

http:/www.vtu.lt/english/editions

\title{
THE VIBROACOUSTICAL INDEXES OF THE INTERNAL COMBUSTION ENGINE WHEN THE MASS OF THE DETAILS OF THE CRANKSHAFT DEVICE VARIES
}

\author{
Eimantas Juodzevičius', Valdas Valiūnas ${ }^{2}$ \\ Department of Automobile Transport, Vilnius Gediminas Technical University; \\ J. Basanavičiaus g. 28, LT- 2009 Vilnius, Lithuania, Tel. (22) 603-697 \\ Received 200205 03; accepted 20020617
}

\begin{abstract}
The vibroacoustical indexes of the internal combustion engine retrogress when the mass of the details of the crankshaft device increases. The blatancy of sound pressure, oscillation speed, the whole engine increase very fractionally when mass increases from 0,5 to $16,0 \mathrm{~g}$. This phenomenon increases loads which affect the principal bearings of the crankshaft as well as the uneven distribution of the loads.
\end{abstract}

Keywords: intemal - combustion engine, crankshaft device, vibroacoustical, sound pressure, unbalanced moment, speed oscillation, actual mass.

\section{Introduction}

Currently either in military or in civil cars the compactness, ergonomicity, comfort of the arrangement of the principal assemblies in the driver's workplace, the passengers' (crew's) sitting (arrangement) places are very topical. The above-mentioned qualities will not be fully implemented; it will be difficult to achieve good results without the positive principal assemblies of the vehicles the engine, the gear - box, the vibroacoustical indexes of the reducer [1].

In the article we will present the description, results and conclusions of the vibroacoustical testing of the principal car assembly - the engine. During the testing the attention will be focused on two references - the potentials of the vibroacoustical equipment and the influence of the mass fluctuation of the details of the crankshaft device (CD) of the internal - combustion engine (ICE) on the vibroacoustical indexes of the engine. Therefore, the purpose of the testing is to determine in the presence of what minimum extra mass the unbalance moment begins to increase and what impact it has on the noise and vibration of the engine.

The literary sources [2-4,] widely describe vibroacoustical testings of base engines to which extra units and systems are fixed in reverberal cells. The purpose of the latter is to determine what impact the vibroacoustical indexes of these elements has on the total noise level transmitted by the engine, also what location of the fixing of the units to select designing new engines. In the described

'E-mail: jeimantas@one.lt

'E-mail: alvydas.pikunas@ti.vtu.lt testings, in our case too, acoustical testing has been used by the automotive industry from the beginning for solving noise and vibration problems. The measurement procedures have been refined in the course of time from the simple use of ear to computer assisted subjective and objective measurements. For example, in objective testing the sound pressure level at a single measurement position is now frequently supplemented by sound power determination [2, 3]. In 1990 Bruel and Kjaer introduced a new measurement technique (a complex) [4] entitled Spatial Transformation of Sound Fields (STSF) which enables the complete description of the sound field of a source to be obtained within a given solid angle. In the automotive industry in particular, STSF has been implemented in testing a wide variety of sources such as:

- engines in various test cells;

- whole vehicles (stationary and on dynam- ometers);

- tyres mounted on vehicles on dynamometers;

- gearboxes on test stands;

- vehicles in wind tunnels.

\section{The Working Schedules of the Engine and Vibro- acoustical References}

While creating, constructing $C D$ details, improving their production technology, the dimensions of the engines diminish and the mass of the $\mathrm{CD}$ details increases because of the use of more resistant steel of greater density $[2,4]$. The same thing happens with the mass of the details of the maintenance measurements as well. Therefore, arranging such details in the mechanism of the engine on the crankpin of each crankshaft unbalanced masses appear, their size is equal to the sum of the difference of 
maintenance and nominal measurement masses of improved and standard $C D$ details, pistons, their sets of rims and connecting - rod linings. Because of each such mass, while the engine is working an extra unbalanced moment emerges, which for V-8 engine equals [2] to:

$$
\mathrm{M}=3,162 \cdot \mathrm{r} \cdot 1 \cdot \omega^{2} \cdot \Delta \mathrm{m}_{\mathrm{i}}
$$

$\mathrm{r}$ - the radius of a crankshaft, $m ; 1$ - the distance between the two adjacent crankpins, $m ; \omega$ - the speed of turning of a crankshaft, $\mathrm{s}^{-1}, \Delta \mathrm{m}_{\mathrm{i}}$ - unbalance mass, $\mathrm{g}$.

It is evident that the increase of the masses of $\mathrm{CD}$ details and the growth of the unbalanced moment connected with it increase vibration and noise levels [5]. How and how much they increase them we will review on V-8 engine, its working capacity is 4,251 . But primarily several advance remarks.

First. Extra unbalance moment affects the engine, very generally, at $n / 60$ frequency ( $n$ - the frequency of turning of a crankshaft), and given namely this frequency, it causes vibration and noise [6]. When the tested engine works on a test stand, two of its own vertical oscillation frequencies prevail. They are equal to 16,7 and $29,6 \mathrm{~Hz}$. They correspond to the resonant frequencies of the turning crankshaft, equal to 1000 and $1600 \mathrm{~min}^{-1}$. Thus, the conclusion is: you cannot estimate the influence of extra unbalanced masses in these schedules. Therefore, for the testing the schedules were selected that are remote enough from the resonant ones, namely: 2400 and $3000 \mathrm{~min}^{-1}$, or 40 and $50 \mathrm{~Hz}$. Such frequency values are selected also due to the fact that they are equal to the corresponding average geometric values of trio octave bands [6].

Second. The vibroacoustical indexes of the engine working at $n / 60$ frequency are influenced by the schedule of load, the relict unbalance values of transmission turning details as well as the eccentricity of the latter with the turning axis of the crankshaft. This effect could be rejected yet, if we tested noise and vibration subject to the unbalance details of the $C D$ when the engine works on idle running [7]. Thus, confining oneself to 40 and $50 \mathrm{~Hz}$ frequency as well as to no-load using vibroacoustical equipment it is possible to get reliable, without errors, values of the vibroacoustical data of the engine based on extra masses and moments [6].

Third. The level of the influence of the increase of the mass of $C D$ details on the vibration of the engine can be assessed either according to the oscillation speed or according to the variation of acceleration spectra. But, as demonstrated by the experience, the best results are obtained according to the oscillation speed [5-7].

\section{Measurement Equipment}

The measurements were performed using the equipment made by Danish firm Bruel \& Kjaer in 1991 - 1993. The equipment consisted of:

- $\quad$ Sound Intensity Analyzer Type 2032;
- Display Type 4715;

- $\quad$ Sound Intensity Probe Type 3519;

- $\quad$ Remote Control Desk Type ZH 0250;

- Telltale Package WB 0845;

- Graphic Literal Printer Type 2318.

This system constitutes keyboard frequency analyzer that measures sound intensity and sound pressure levels in mono-octave and trio octave bands, when the frequency is $3,2-10 \mathrm{kHz}$ (sound intensity) and 1,6-20 $\mathrm{kHz}$ (sound pressure). The results are presented on the 4715-display screen according to real - time analysis principle, where inter alia array direction of sound intensity is indicated and Printer 2318 prints the results.

\section{Measurement Procedure}

For good results well - defined measurement conditions are required. Information about the references used, environmental conditions, the grid size, the presence of a reflecting plane, etc. is put into a measurement set - up (register) and stored with the measured data.

Before performing any calculation the data are inspected and validated using the functions available in the software to compare the directly measured data with the results derived from the description of the sound field established by STSF program. The comparison is necessary here in order to obtain accurate values of references [2].

If necessary, data can be re-measured and replaced. There will always be some discrepancy between the directly measured and the calculated spectra due to non stationarity of the source during the measurement at low frequencies, the use of an insufficient set of references, or uncorrelated background noise. These errors are not estimated during this measurement.

\section{Measurement Results}

In the process of the engine testing these references were rated.

When the extra mass of $C D$ details varies from zero to $5,0 \mathrm{~g}$ within the pale of component 40 and $50 \mathrm{~Hz}$ frequency of oscillation speed spectra, general levels of this speed flow $\mathrm{L}_{\mathrm{v}}$ practically do not fluctuate, that is, $\Delta \mathrm{m}_{\mathrm{i}}=0 \div 5,0 \mathrm{~g}$ does not have any impact on the vibration of the engine. However, during the experiment further increasing this mass (to approximately $16,0 \mathrm{~g}$ ) and spectra component, general levels of oscillation speed increase sharply. In comparison, the components in the telltale points increase 9 -$20 \mathrm{~dB}$, general levels of speed oscillation $-4 \div 16 \mathrm{~dB}$. Graphic expression is presented in Fig 1.

The view with sound pressures (of blatancy) is approximately the same. When $\Delta \mathrm{m}_{\mathrm{i}}=0 \div 5,0 \mathrm{~g}$ band sound pressure levels $\left(L_{p}\right)$ over sound and frequency $n / 60$ do not vary. But raising $\Delta m_{i}$ to $16,0 \mathrm{~g}$, the first increases $6-13$ $\mathrm{dB}$, the second - to $1-4 \mathrm{~dB}$ (Fig 2).

The obtained summarized references are also illustra- 


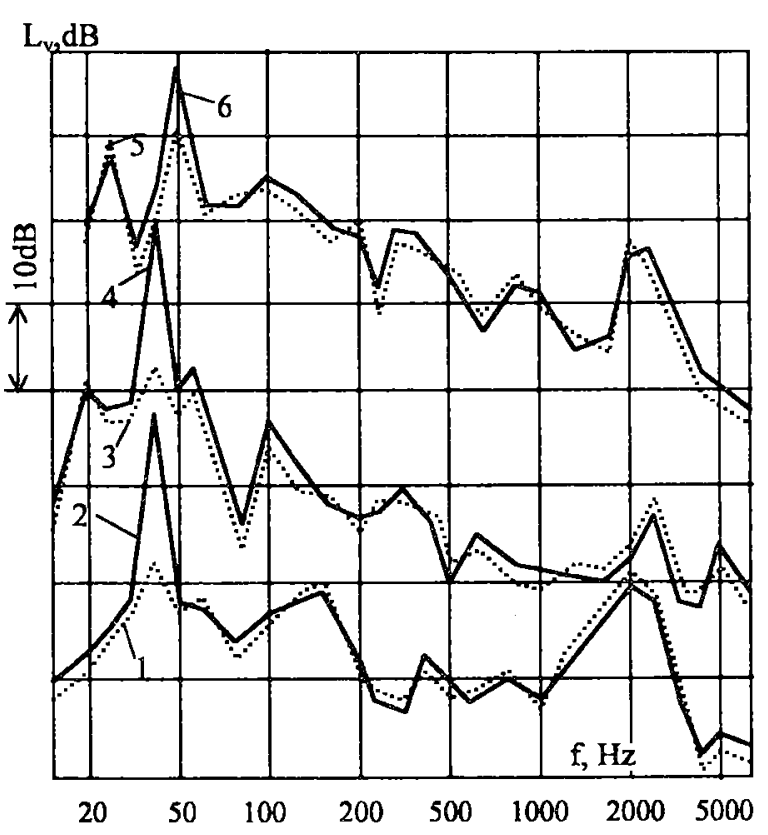

Fig 1. The spectra of the speed oscillation: 1 and $2-$ in axial direction respectively, when $\Delta \mathrm{m}_{\mathrm{i}}=0$ and $\Delta \mathrm{m}_{\mathrm{i}}=16,0 \mathrm{~g} ; 3$ and $4-$ in cross direction, when $\Delta \mathrm{m}_{\mathrm{i}}=0$ and $\Delta \mathrm{m}_{\mathrm{i}}=16,0 \mathrm{~g} ; 5$ and 6 in vertical direction, when $\Delta \mathrm{m}_{\mathrm{i}}=0$ and $\Delta \mathrm{m}_{\mathrm{i}}=16,0 \mathrm{~g}(1,2$ and

3,4 the coordinate beginning of the spectra subject to the spectra 5 and 6 is run 30 and $18 \mathrm{~dB}$ respectively)

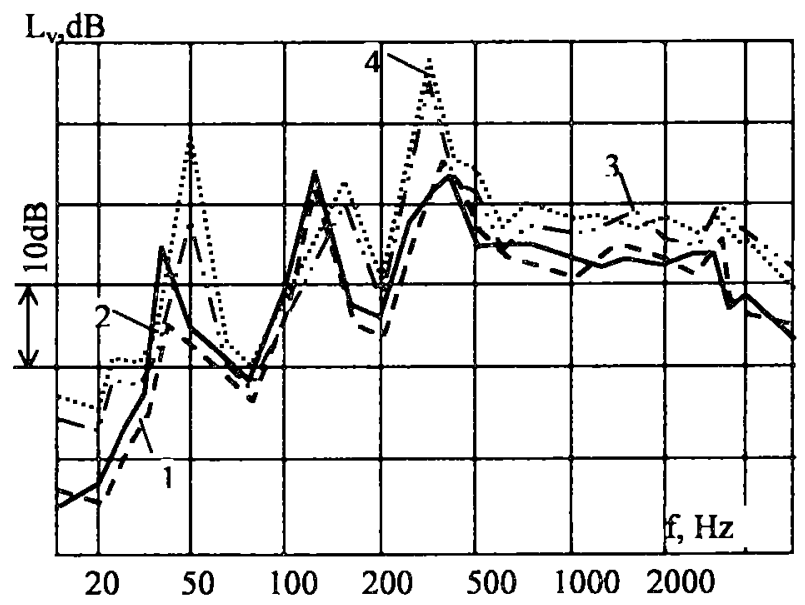

Fig 2. The spectra of the sound pressure: 1 - when $\Delta m_{i}=0$ and $2400 \mathrm{~min}^{-1}: 2-$ when $\Delta \mathrm{m}_{\mathrm{i}}=16,0 \mathrm{~g}$ and $2400 \mathrm{~min}^{-1}$; $3-$ when $\Delta \mathrm{m}_{\mathrm{i}}=0$ and $3000 \mathrm{~min}^{-1} ; 4-$ when $\Delta \mathrm{m}_{\mathrm{i}}=16,0 \mathrm{~g}$ and $3000 \mathrm{~min}^{-1}$

ted in Fig 3 and 4, where the results of the experimental testing of the influence of the mass of CD details on the oscillation speed and sound pressure when the engine works at $n / 60$ frequency are presented.

It is obvious that there are two specific fields in the graphs presented in these diagrams. In the first field, where $\Delta \mathrm{m}_{\mathrm{i}}$ varies from zero to $5,0 \mathrm{~g}$, the vibration in the cross and axial directions as well as the noise of the engine do not depend on this mass. But in the second field, where the

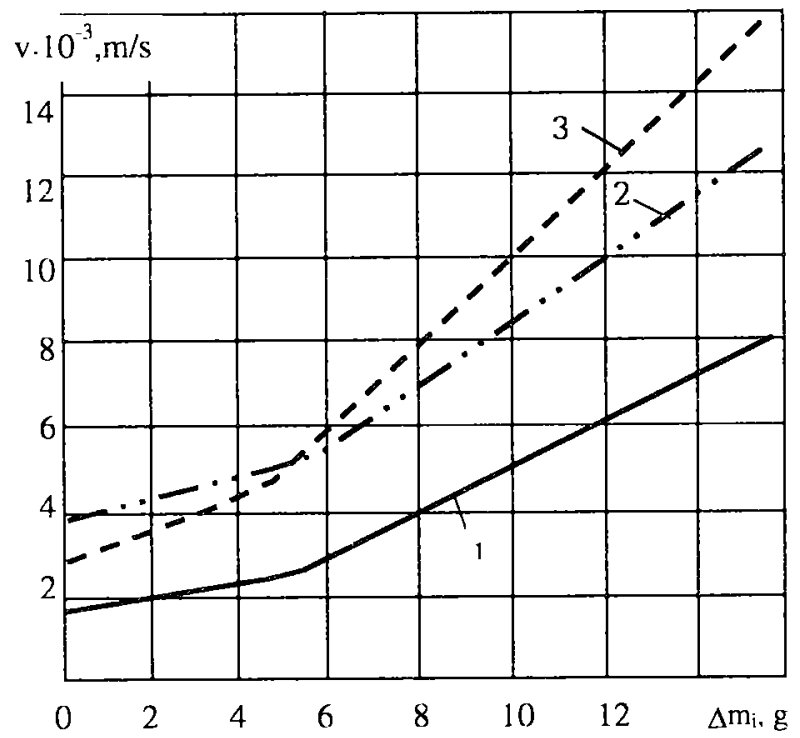

Fig 3. The dependence of the speed oscillation on $\Delta \mathrm{m}_{\mathrm{i}}$ : 1 and 2 - in vertical direction given 2400 and $3000 \mathrm{~min}^{-1}$; 3 - in axial direction given $2400 \mathrm{~min}^{-1}$

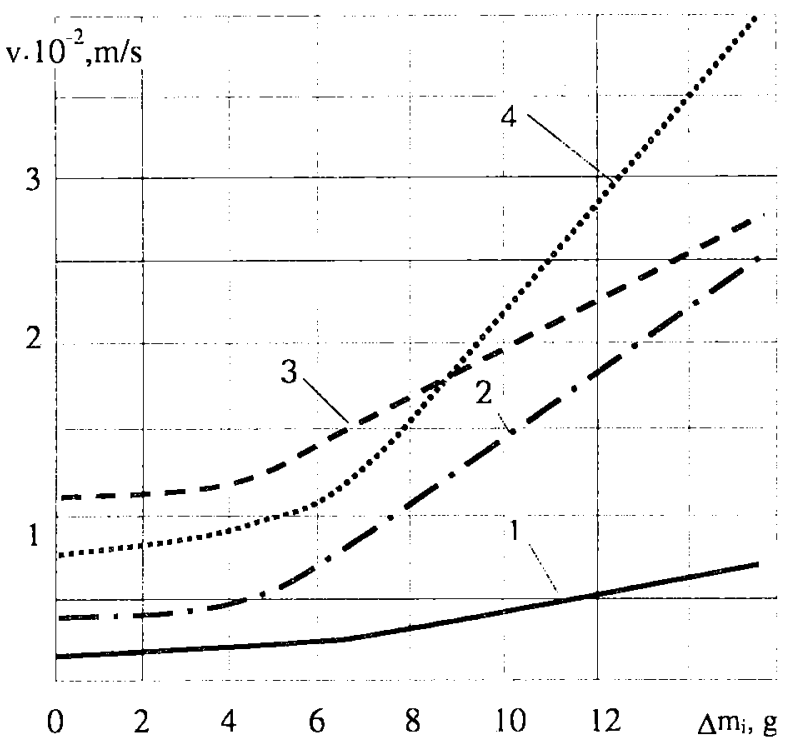

Fig 4. The dependence of the sound pressure on $\Delta \mathrm{m}_{\mathrm{i}}$ : 1 and $2-$ in axial and cross directions given $2400 \mathrm{~min}^{-1}$; 3 and 4 - in vertical direction given $3000 \mathrm{~min}^{-1}$ and the sensor setting in various points

extra unbalanced mass increases to approximately $16,0 \mathrm{~g}$, the clear increase of the vibroacoustical indexes of the engine is observed.

Besides, the functions are fully approximated in the straight lines going through the beginning of the coordinate. This process could be explained in this way. The unbalanced mass increasing, the moment increases agreeably to the linear dependence. But as the turning speed of the crankshaft does not change, it rises from the straight line going through the beginning of the coordinate. It is presented in Figs 5 and 6. 


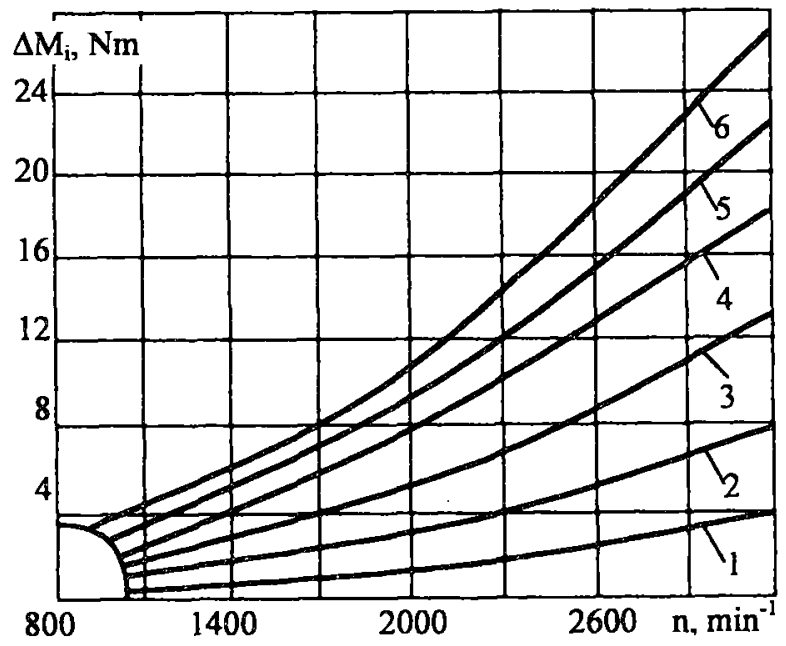

Fig 5. The dependence of the extra unbalance moment on the turning speed of a crankshaft: $1,2,3,4,5,6$ - respectively, when $\Delta \mathrm{m}_{\mathrm{i}}$ is equal tos 2,$3 ; 5,0 ; 6,9 ; 10,2 ; 13,5 ; 16,0 \mathrm{~g}$

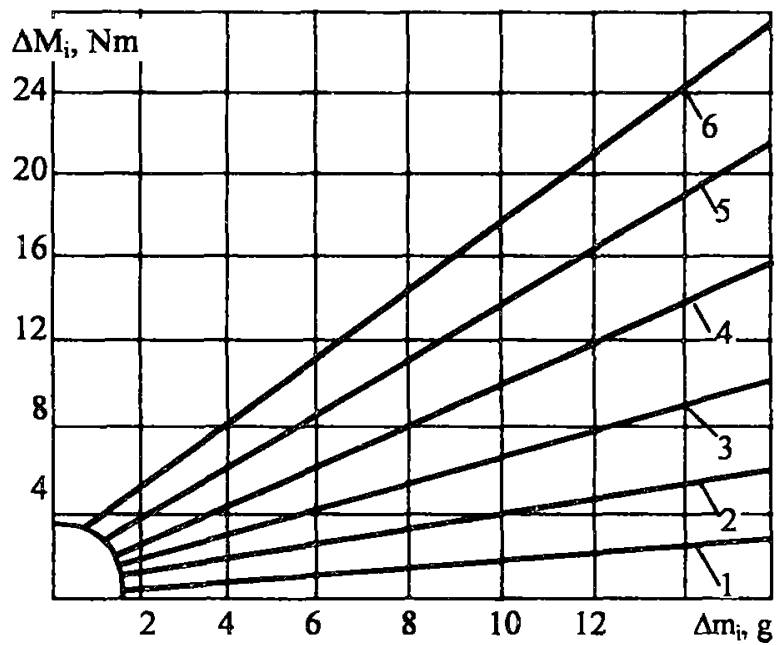

Fig 6. The dependence of the extra unbalance moment on $\Delta \mathrm{m}_{\mathrm{i}}: 1,2,3,4,5,6$ - respectively, when the revolutions of a crankshaft are equal to $1000,1500,1900,2400,2800$, $3200 \mathrm{~min}^{-1}$

$\Delta m_{i}$ being fixed, the moment from this mass varies subject to the frequency of the turning of the crankshaft according to the parabolic graph. Thus, the conclusion can be made that either $\Delta \mathrm{m}_{\mathrm{i}}$ or the frequency of the turning of the crankshaft increasing as well as both the references increasing at the same time, the unbalanced moment rises. This phenomenon increases loads which affect the principal bearings of the crankshaft as well as the uneven distribution of the loads.

The vibroacoustical indexes of the engine vary approximately according to the same algorithm. For example, rising from 0 to $5,0 \mathrm{~g}$, the moment increases only $0,79-8,05 \mathrm{Nm}$. The loads caused by this moment do not have any substantial impact on the key bearings. In any case - much less than the loads caused by other factors (gas and inert forces, the distribution of the uneven and discreet adjusting fits in the bearings, the deflection of the forms of bearings and mutual position, the hog of the crankshaft under the circumstances of repairs or production, the variation of all these references with the schedules of the speed of the engine and load, the thermal schedule, etc.). In the second field, where $\Delta m_{i}$ is raised to $16,0 \mathrm{~g}$ load affecting the bearings and their uneven distribution caused by the unbalanced moment in this field is bigger than the above mentioned factors [8]. For instance, in Fig 5 we can see that the unbalanced moment in this field varies from 1,18 to $27,42 \mathrm{Nm}$. The loads affecting the bearings as well as their uneven distribution increase respectively. It is clear that this is the reason for the increase of the vibration and sound pressure of the engines. Besides, the temperature of the bottom principal inserts rises $4-7 \mathrm{~K}$, mechanic wastage $-4,1-5,3 \%$ [1]. We can maintain that the necks of the crankshaft scuff faster; the coefficient of their abrasion unevenness becomes higher.

\section{The Utilization of the Vibroacoustical Measurements in the Calculations}

Such is the "physics" of the phenomena related to the emergence of the unbalanced masses and moments. For practical purposes it is also important to know how to utilize calculations in order to determine vibroacoustical performances. There is such a possibility.

For example, for the engine under the investigation, in the field, where $\Delta \mathrm{m}_{\mathrm{i}}$ is more than $5,0 \mathrm{~g}$, the values of oscillation speed and sound pressure can be calculated according to the formulas that differ in the coefficients $\mathrm{K}_{v}$ and $K_{p}$ (the coefficients of the oscillation speed and sound pressure respectively). These coefficients are multiplied by the component of the moment $\mathrm{K}_{\mathrm{i}}$ :

$$
\mathrm{K}_{\mathrm{i}}=0,0347 \cdot \mathrm{r} \cdot 1 \cdot \mathrm{n}^{2} \cdot \Delta \mathrm{m}_{\mathrm{i}}
$$

All the values are known except the coefficients $K_{v}$ and $\mathrm{K}_{\mathrm{p}}$. They can be determined by test. It is done in this way.

The values of the turning speed of the crankshaft $n_{0}$ and $\Delta \mathrm{m}_{\mathrm{i}}$ being fixed and the value of $\Delta \mathrm{m}_{\mathrm{i}}$ corresponding to the maximum $\Delta \mathrm{m}_{\text {imax }}$ in the process of the testing, the oscillation speed $V_{0}$ and sound pressure of the engine $p_{0}$ are measured. Afterwards these values are allotted to the products:

$$
\mathrm{K}_{\mathrm{i}}=0,0347 \cdot \mathrm{r} \cdot 1 \cdot \mathrm{n}^{2} \cdot \Delta \mathrm{m}_{\text {imax }} \text {. }
$$

The obtained values will be the wanted coefficients.

However, all this can be fulfilled on condition that the vibration and noise of the engine, $n_{0}$ and $\Delta m_{i}$ being fixed, depend on the location of the sensors and their measurement direction. It means that the coefficients $\mathrm{K}_{\mathrm{v}}$ and $\mathrm{K}_{\mathrm{p}}$ belong, too. Therefore, all the measurements must be performed at the same points.

One more circumstance may be mentioned. 
Unbalanced masses $\Delta \mathrm{m}_{\mathrm{i}}$ emerge not only producing or repairing $C D$ details. Their equivalent emerges exploiting the engines, too: scales and resins resolve on pistons and their rims and attrition products and dirt - in dirt assemblents of crankpins. The test on the experimental engine shows that after resource output until major repairs these extra masses can reach $0,42-1,86$ and $1,15-3,65 \mathrm{~g}$ respectively. These masses are summed up with $\Delta \mathrm{m}_{\mathrm{i}}$ and at the same time raise the vibration and noise of the engine [9]. There is an example described in the literature when in 16 tested engines of this type the levels of their oscillation speed and sound pressure, as a result of scales and resins, increased $3-8$, and as a result of sludge dirt in the assemblents $-2-5 \mathrm{~dB}$.

With the help of repairs not all negative phenomena can be eliminated. The deviations of the actual mass of maintenance pistons, their rims and inserts of connecting - rod bearings, nibs and connecting - rods of pistons, which are assembled into 4,25 and 6,0 I V-8 Type engines, are 2-18 times greater than the permissible variation. Therefore, the values of the unbalanced masses $\Delta \mathrm{m}_{\mathrm{i}}$ after major repairs can reach 7,8-18,1 g [9]. These masses can cause the fluctuations described above (if there are any frequencies).

\section{Conclusions}

1. Revolutions being equal and extra mass changing, the levels of sound pressure vertically are greater than in axial and transverse directions.

2. The levels of sound pressure, oscillation speed, the blatancy of the whole engine increase very fractionally when $\Delta \mathrm{m}_{\mathrm{i}}$ increases from 0 to $5,0 \mathrm{~g}$, but they rise considerably when $\Delta \mathrm{m}_{\mathrm{i}}$ increases to $16,0 \mathrm{~g}$. The unbalanced mass rising further, the vibration and noise of the engine increase proportionally.

3. $\Delta \mathrm{m}_{\mathrm{i}}$ mass increasing, the unbalanced moment of the engine which increases loads that affect the principal bearings of the crankshaft as well as the uneven distribution of the loads, increases. When the discreteness of loads emerges, the vibration of the engine increases.

4. The levels of oscillation speed which are greater in transverse direction, where the area of the sent out noise is the greatest, the noise of the engine increases.

\section{References}

1. Watkinson P. S. Sound intensity measurement. The shock and vibration digest, Vol 17, No 5, January 1997.

2. Hald J. STSF unique technique for scan-based near field acoustic holography without restriction on coherence. Bruel \& Kjaer Technical Review, 1991, No 2. 40 p.

3. Juodzevičius $E$. The utilization of noise produced by means of transport for their diagnostics. Transport Engineering (Transportas), 2001, XVI t., No 6, p $234-239$.

4. Willows U., Hald J. and Ginn K. B. Acoustical testing of a diesel engine using STSF. Nærum: Bruel \& Kjaer, 1992. 32 p.

5. Gonzalez A., Elliot S. J. and etc. Minimisation of the maximum error signal in active control. In: Proceedings of the International Conference on Acoustics, Speech and Signal Processing (ICASSP) 1, 1997, p 1-4.

6. Ripak M., Panachek I. STSF - method of research of noise produced by MV. Automotive industry (Автомобильная промылиленность), 1999, No 3, p 30-33.

7. Bruel P. V. Microphone configurations used in acoustic intensity probes. Senlis. In: Proceedings of 2 nd international congress on acoustic intensity, 1994, p 3-4.

8. E. M. Eibeheiry. Effects of small travel speed variation on active vibration control in modern vehicles. Joumal of Sound and Vibration, Vol 232(5), 2000, p 857-875.

9. Cheng C. C., Shiu J. S. Transient vibration analysis of a high-speed feed drive system. Journal of Sound and Vibration, Vol 239(3), 2001, p 489-504. 